\title{
NORMALITY AND PROPERTIES RELATED TO COMPACTNESS IN HYPERSPACES
}

\section{JAMES KEESLING}

Introduction. Let $X$ be a regular $T_{1}$ topological space and $2^{X}$ the space of all closed nonempty subsets of $X$ with the finite topology [8, Definition 1.7]. In [6] Ivanova has shown that if $X$ is a noncompact ordinal space, then $2^{X}$ is nonnormal. In this paper we give a new proof of this fact. This result is then used to show that several properties of $2^{x}$ are equivalent to the compactness of $X$. It is not known if the normality of $2^{X}$ is equivalent to the compactness of $X$. There are some partial results in this direction though. The paracompactness of $2^{X}$ is shown to be equivalent to the compactness of $X$ and the normality of $2^{2^{x}}$ is also shown to be equivalent to the compactness of $X$. In the last part of the paper some properties related to the countable compactness of $2^{X}$ are investigated.

Notation. Because of our assumptions on $X, \hat{X}=\{\{x\}: x \in X\}$ is a closed subset of $2^{X}$ homeomorphic to $X$. The set $\mathcal{F}_{n}(X)=\{F \subset X: F$ has at most $n$ points $\}$ is also closed. Furthermore, the space $2^{X}$ is Hausdorff. For notation and further basic results on hyperspaces see [7] or [8]. In particular we use $\left\langle U_{1}, \cdots, U_{n}\right\rangle=\left\{A \in 2^{X}: A \subset \bigcup_{i=1}^{n} U_{i}\right.$ and $A \cap U_{i} \neq \varnothing$ for all $\left.i\right\}$. If each $U_{i}$ is open in $X$, then $\left\langle U_{1}, \cdots, U_{n}\right\rangle$ is open in $2^{X}$ and the set of such sets in $2^{X}$ forms a basis for $2^{X}$. By considering such basic open sets it is clear that the set $\mathcal{F}(X)$ of finite subsets of $X$ is dense in $2^{X}$. We denote the cardinality of a set $Z$ by $|Z|$.

1. The hyperspace of a discrete space. It follows from Ivanova's result [6], that the hyperspace of an infinite discrete space is nonnormal. We give a new proof of this result based on the following lemma.

Lemma. If $X$ is an infinite discrete space of cardinality $\sigma$, then $2^{X}$ has a dense subset of cardinality $\sigma$ and a closed discrete subset of cardinality $2^{\sigma}$.

Proof. The set $F(X)$ of finite subsets of $X$ is dense in $2^{X}$ with $|\mathfrak{F}(X)|=|X|=\sigma$. To prove the last statement, let $X=X_{1} \cup X_{2}$ be a disjoint union with $\left|X_{i}\right|=\sigma$. Let $f_{i}: X \rightarrow X_{i}$ be a bijection for each $i$ and let $F: 2^{X} \rightarrow 2^{X}$ be defined by $F(A)=f_{1}(A) \cup f_{2}(X-A)$. Let $Q=\{F(A): A \subset X\}$. Now for $A \subset X$, letting $\mathcal{u}=2^{F(A)}$ which is open in $2^{x}$, we have $u \cap a=\{F(A)\}$. Thus $Q$ is discrete. Now if $B \in 2^{x}$ and

Received by the editors February 14, 1969. 
$B \notin a$, then either $f_{1}^{-1}\left(B \cap X_{1}\right) \cup f_{2}^{-1}\left(B \cap X_{2}\right) \neq X$ or $f_{1}^{-1}\left(B \cap X_{1}\right)$ $\cap f_{2}^{-1}\left(B \cap X_{2}\right) \neq \varnothing$. In either case one can easily show that $B$ is not in the closure of $Q$. Thus $Q$ is closed and discrete. It is clearly of cardinality $2^{\sigma}$ since $F$ is an injection.

Theorem 1. If $X$ is infinite and discrete, then $2^{X}$ is nonnormal.

Proof. Since $2^{x}$ has a dense subset of cardinality $\sigma$, there are at most $2^{\sigma}$ continuous real valued functions on $2^{x}$. But the existence of $a$ in the proof of the lemma says that if $2^{X}$ is normal, then by extending each characteristic function of each subset of $Q$ to all of $2^{x}$ (as real valued functions), there must be at least $2^{2^{*}}$ continuous real valued functions on $2^{x}$. Thus we cannot have $2^{x}$ normal.

CoROllary. If $2^{X}$ is normal, then $X$ is countably compact.

Proof. If $X$ is not countably compact, then there is a closed discrete subset $D$ in $X$. But then $2^{D}$ is a closed subspace of $2^{X}$. Since $2^{D}$ is nonnormal, $2^{X}$ is nonnormal.

2. Compactness of the hyperspace. We now show that several properties related to compactness are equivalent to compactness in the hyperspace.

DEFinition. A topological space is said to be paracompact if every open cover has a locally finite refinement [2, p. 162]. It is said to be metacompact if each open cover has a point finite open refinement [2, p. 229]. It is meta-Lindelöf (countably metacompact in [1]) if each open cover has a point countable open refinement.

Lemma. If $N$ is the integers with discrete topology, then $2^{N}$ is not meta-Lindelöf.

Proof. Since $2^{N}$ is separable, if it is meta-Lindelöf it is Lindelöf. However, $2^{N}$ is regular [8, Theorem 4.9]. Thus if $2^{N}$ is meta-Lindelöf, then $2^{N}$ is normal contradicting Theorem 1 .

THEOREM 2. The following are equivalent:

(a) $X$ is compact.

(b) $2^{X}$ is compact,

(c) $2^{x}$ is Lindelöf,

(d) $2^{X}$ is paracompact,

(e) $2^{X}$ is metacompact, and

(f) $2^{x}$ is meta-Lindelöf.

Proof. It is well known that (a) is equivalent to (b) $[8$, Theorem 4.2 ]. It will be sufficient to show that (f) implies (a). If $2^{x}$ is meta- 
Lindelöf, then so is $X$ since $\hat{X}$ is a closed subset of $2^{x}$. By the corollary to Theorem 1 we must have that $X$ is also countably compact. Thus $X$ is meta-Lindelöf and countably compact, hence compact [1].

3. Normality of the hyperspace of the hyperspace. We will now need the fact that if $X$ is a noncompact well ordered space, then $2^{X}$ is nonnormal. This result is due to Ivanova [6] and for completeness we now include a proof.

THEOREM 3. If $X$ is a noncompact ordinal space, then $2^{X}$ is nonnormal.

Proof. Let $X=[0, \alpha)=\{\gamma: \gamma$ is an ordinal less than $\alpha\}$. By Theorem 1 we need only consider the case that $X$ is countably compact. In this case there is no countable cofinal sequence of ordinals in $[0, \alpha)$. We now give Ivanova's proof for this case. Let $X_{\sigma}=[\sigma, \alpha)$ for each $\sigma$ less than $\alpha$. Then $\mathcal{F}=\left\{X_{\sigma}\right\}$ is a closed subset of $2^{X}$ and $\mathcal{F}$ and $\hat{X}$ are disjoint in $2^{X}$. If $2^{x}$ were normal, there would be an open set $u$ containing $\mathcal{F}$ whose closure misses $\hat{X}$. Let $X_{\sigma} \in\left\langle U_{1}^{\sigma}, \cdots, U_{n}^{\sigma}\right\rangle \subset \mathcal{u}$ for each $\sigma$ where $U_{i}^{\sigma}$ is open in $X$. Let $\lambda_{i}^{0} \in U_{i}^{0} \cap X_{0}$ and $\sigma_{1}=\max \left\{\lambda_{i}^{0}\right\}$. Then $\left\{\lambda_{1}^{0}, \cdots, \lambda_{n}^{0}\right\} \in\left\langle U_{1}^{0}, \cdots, U_{n}^{0}\right\rangle$. Now let $\lambda_{i}^{1} \in U_{i}^{\sigma_{1}} \cap X_{\sigma_{1}}$ and $\sigma_{2}=\max \left\{\lambda_{i}^{1}\right\}$. Then $\left\{\lambda_{1}^{1}, \cdots, \lambda_{n}^{1}\right\} \in\left\langle U_{1}^{\sigma_{1}}, \cdots, U_{n}^{\sigma_{1}}\right\rangle$. Continuing this process inductively we get an increasing sequence of ordinals $\left\{\sigma_{j}\right\}$ in $[0, \alpha)$ and a countable collection of finite subsets of $X$, $\left\{\lambda_{i}^{\prime}: i=1, \cdots, n_{j}\right\}$ with the property that $\left\{\lambda_{i}^{j}\right\} \in \mathcal{U}$ for each $j$ and $\left\{\lambda_{i}^{j}: i=1, \cdots, n_{j}\right\} \subset\left[\sigma_{j}, \sigma_{j+1}\right]$. Let $\sigma=\sup \left\{\sigma_{j}\right\}$ which is less than $\alpha$. Then in $2^{x}$ the singleton set $\{\sigma\}$ is in the closure of $u$ since the sequence $\left\{\left\{\lambda_{i}^{\prime}: i=1, \cdots, n_{j}\right\}: j=1,2,3, \cdots\right\}$ converges to $\{\sigma\}$. Thus $\mathcal{F}$ and $\hat{X}$ cannot be separated in $2^{x}$.

Theorem 4. If $2^{2^{X}}$ is normal, then $X$ is compact.

Proof. We will show that there is a limit ordinal $\alpha$ such that $[0, \alpha)$ can be imbedded as a closed subset of $2^{x}$ if $X$ is not compact. The theorem will then follow from Theorem 3 since $2^{(0, \alpha)}$ will then be a closed subspace of $2^{2^{X}}$. Now suppose that $X$ is not compact and let $\mathcal{F}$ be a filter base of closed sets with empty intersection. Let us suppose that $|\mathcal{F}|=\sigma$ and that $\sigma$ is the minimum cardinal with the property that there is such a filter base. That is, if $g$ is a collection of closed sets in $X$ with finite intersection property with $|g|$ less than $\sigma$, then the intersection of $g$ is nonempty. Identify $\sigma$ with the first ordinal having that cardinality. Then $[0, \sigma)$ has cardinality $\sigma$ and $[0, \gamma)$ has cardinality less than $\sigma$ for $\gamma$ less than $\sigma$. Then let $\mathcal{F}$ be indexed by $\left\{F_{\alpha}: \alpha<\sigma\right\}$. By transfinite induction we define a sequence $\left\{\alpha_{\gamma}: \gamma<\sigma\right\}$ 
in $[0, \sigma)$ in the following manner. We let $\alpha_{0}=0$. Having defined $\alpha_{\gamma}$ let $\alpha_{\gamma+1}$ be the first $\alpha$ such that $\cap\left\{F_{\alpha}: \alpha \leqq \alpha_{\gamma+1}\right\}$ is properly contained in $\cap\left\{F_{\alpha}: \alpha \leqq \alpha_{\gamma}\right\}$. For $\gamma$ a limit ordinal, let $\alpha_{\gamma}=\sup \left\{\alpha_{\lambda}: \lambda<\gamma\right\}$. It is clear that this process may be continued until $\cap\left\{F_{\alpha_{\gamma}}\right\}=\varnothing$. But this will require using all $\gamma$ 's less than $\sigma$ by the minimality of $\sigma$. Now let $G_{\gamma}=\bigcap\left\{F_{\alpha}: \alpha \leqq \alpha_{\gamma}\right\}$. Then $G_{\gamma}$ is closed and $G_{\gamma} \neq \varnothing$ for all $\gamma$. Thus $G_{\gamma} \in 2^{x}$. We now show that $\left\{G_{\gamma}\right\}$ is our required set.

Claim. The set $\left\{G_{\gamma}: \gamma<\sigma\right\}$ is a closed subset of $2^{x}$.

Proof of claim. If $F$ is an element of $2^{x}$ and not of $\left\{G_{\gamma}\right\}$, then if $F$ is not contained in any $G_{\gamma}$ let $\mathcal{U}=2^{x}-2^{G_{0}}$. Then $\mathcal{U}$ is an open set in $2^{x}$ containing $F$ and containing no element of $\left\{G_{\gamma}\right\}$. Now if $F \subset G_{\gamma}$ for some $\gamma$, then let $A=\bigcap\left\{G_{\gamma}: F \subset G_{\gamma}\right\}$. Then $A=G_{\lambda}$ where $\lambda=\sup \left\{\gamma: F \subset G_{\gamma}\right\}$ and $F$ is not contained in $G_{\lambda+1}$. Let $x \in G_{\lambda}-F$ and let $\mathcal{u}=2^{X-|x|}-2^{G_{\lambda+1}}$. Then $F \in \mathcal{u}$ with $\mathcal{u}$ open and $\mathcal{u} \cap\left\{G_{\gamma}\right\}=\varnothing$.

Claim. If $f(\gamma)=G_{\gamma}$, then $f$ is a homeomorphism from $[0, \sigma)$ onto $\left\{G_{\gamma}\right\}$.

Proof of ClaIm. Clearly $f$ is one to one and onto. We now show that $f$ is bicontinuous. Let $G_{\lambda} \in\left\{G_{\gamma}\right\}$ and $x \in G_{\alpha}-G_{\lambda+1}$ for any $\alpha<\lambda$. Then if $u=2^{X-\{x\}}-2^{G_{\lambda+1}}$, then $f([\alpha+1, \lambda])=u \cap\left\{G_{\gamma}\right\}$. Thus $f^{-1}$ is continuous. Now let $\lambda \in[0, \sigma)$ and let $G_{\lambda} \in\left\langle U_{1}, \cdots, U_{n}\right\rangle$. Since $G_{\lambda+1}$ is closed and properly contained in $G_{\lambda}$ we may assume that $\left\langle U_{1}, \cdots, U_{n}\right\rangle \cap\left\{G_{\gamma}\right\}=\left\{G_{\gamma}: G_{\gamma} \supset G_{\lambda}\right.$ and $\left.G_{\gamma} \subset \cup_{i=1}^{n} U_{i}\right\}$ as a typical basic neighborhood of $G_{\lambda}$ in $\left\{G_{\gamma}\right\}$. Now suppose that the set $\left\{\gamma: G_{\gamma} \subset \cup_{i=1}^{n} U_{i}\right.$ and $\left.G_{\gamma} \supset G_{\lambda}\right\}$ does not contain $\lambda$ in its interior. Then there is a set $\left\{\gamma_{\alpha}\right\}$ such that (1) $\gamma_{\alpha}<\lambda$ for all $\alpha$, (2) $\sup \left\{\gamma_{\alpha}\right\}=\lambda$, and (3) $G_{\gamma_{\alpha}}$ is not contained in $\bigcup_{i=1}^{n} U_{i}$ for each $\alpha$. Now $\left\{G_{\gamma_{\alpha}}-U_{i=1}^{n} U_{i}\right\}$ has the finite intersection property and empty intersection. Thus $\left\{\gamma_{\alpha}\right\}$ has cardinality $\sigma$ and $\left\{\gamma_{\alpha}\right\}$ is cofinal in $[0, \sigma)$, contradicting the fact that $\sup \left\{\gamma_{\alpha}\right\}=\lambda<\sigma$. Thus $\lambda$ is in the interior of $\left\{\gamma: G_{\gamma} \subset \cup_{i=1}^{n} U_{i}\right.$ and $\left.G_{\gamma} \supset G_{\lambda}\right\}$ and $f$ is continuous. Thus $f$ is a homeomorphism. The proof of Theorem 4 is now complete.

4. Countable compactness and hyperspaces. We show that $2^{x}$ can be countably compact and noncompact. We also give an example of a countably compact completely regular space $X$ such that $2^{X}$ is not pseudocompact.

Definition. A space $X$ is pseudocompact if each real valued continuous function is bounded. A space is strongly countably compact if each countable set has compact closure.

If a space is strongly countably compact, then it is countably compact. If it is countably compact, then it is pseudocompact. Neither of these implications is reversible in general. The property of being strongly countably compact has very interesting properties. 
It is productive, closed hereditary, and preserved under continuous transformation. The next theorem gives an additional result for this property. The following lemma is well known [9]. (Compare [5, 6.5 (IV), p. 86].)

Lemma. If $X$ is normal and $\left\{F_{1}, \cdots, F_{k}\right\}$ is a finite collection of closed subsets of $X$, then $\bigcap_{i=1}^{k} \mathrm{Cl}_{\beta X} F_{i}=\mathrm{Cl}_{\beta X}\left[\bigcap_{i=1}^{k} F_{i}\right]$.

Proof. We will show this for two closed sets $F_{1}$ and $F_{2}$ in $X$. The lemma will then follow by induction. Clearly, $\mathrm{Cl}_{\beta X}\left(F_{1} \cap F_{2}\right) \subset \mathrm{Cl}_{\beta X} F_{1}$ $\cap \mathrm{Cl}_{\beta X} F_{2}$. Suppose that $x$ is an element of $\mathrm{Cl}_{\beta X} F_{1} \cap \mathrm{Cl}_{\beta X} F_{2}$ and not of $\mathrm{Cl}_{\beta X}\left(F_{1} \cap F_{2}\right)$. Let $x \in V$ with $V$ open in $\beta X$ such that $\mathrm{Cl}_{\beta X} V$ $\cap \mathrm{Cl}_{\beta X}\left(F_{1} \cap F_{2}\right)=\varnothing$. Then let $A_{i}=F_{i} \cap \mathrm{Cl}_{X} V$ for $i=1$ and $i=2$. Then $x$ is an element of $\mathrm{Cl}_{\beta X} A_{1} \cap \mathrm{Cl}_{\beta X} A_{2}$ since $x \in \mathrm{Cl}_{\beta X} F_{i}$ for each $i$. But $A_{1} \cap A_{2}=\varnothing$ since $\mathrm{Cl}_{X} V \cap\left(F_{1} \cap F_{2}\right)=\varnothing$. By the normality of $X$ we have $\mathrm{Cl}_{\beta X} A_{1} \cap \mathrm{Cl}_{\beta X} A_{2}=\varnothing$, a contradiction. Thus $\mathrm{Cl}_{\beta X}\left(F_{1} \cap F_{2}\right)$ $=\mathrm{Cl}_{\beta X} F_{1} \cap \mathrm{Cl}_{\beta X} F_{2}$.

Lemma. If $X$ is normal and $F: 2^{X} \rightarrow 2^{\beta X}$ is defined by $F(K)=\mathrm{Cl}_{\beta X} K$, then $F$ is an imbedding.

Proof. Suppose that $F(K) \in\left\langle U_{1}, \cdots, U_{n}\right\rangle$ for $K \in 2^{x}$, with each $U_{i}$ open in $\beta X$. Let $\left\{V_{1}, \cdots, V_{n}\right\}$ be a collection of open subsets of $\beta X$ with $F(K) \subset \bigcup_{i=1}^{n} V_{i}, \mathrm{Cl}_{X} V_{i} \subset U_{i}$ for each $i$, and with $F(K)$ $\cap V_{i} \neq \varnothing$ for each $i$. Let $O_{i}=V_{i} \cap X$. One can easily verify that $K \in\left\langle O_{1}, \cdots, O_{n}\right\rangle$ and that, for $L \in 2^{X}$ with $L \in\left\langle O_{1}, \cdots, O_{n}\right\rangle$, $F(L) \in\left\langle U_{1}, \cdots, U_{n}\right\rangle$.

Now suppose that $K \in 2^{x}$ with $K \in\left\langle U_{1}, \cdots, U_{n}\right\rangle$. Then, of course, $K \subset \bigcup_{i=1}^{n} U_{i}$. Thus $K \cap\left(\bigcap_{i=1}^{n}\left(X-U_{i}\right)\right)=\varnothing$. By the foregoing lemma, $\mathrm{Cl}_{\beta X} K \cap\left(\cap_{i=1}^{n}\left(\mathrm{Cl}_{\beta X}\left(X-U_{i}\right)\right)\right)=\varnothing$. If we let $O_{i}=\beta X-\mathrm{Cl}_{\beta X}\left(X-U_{i}\right)$, then the last statement implies that $\mathrm{Cl}_{\beta X} K \subset \mathrm{U}_{i=1}^{n} O_{i}$. Thus $F(K)$ $\in\left\langle O_{1}, \cdots, O_{n}\right\rangle$. But if $F(L) \in\left\langle O_{1}, \cdots, O_{n}\right\rangle$, then $L \in\left\langle U_{1}, \cdots, U_{n}\right\rangle$. Thus $F^{-1}$ is continuous from $F\left(2^{x}\right)$ onto $2^{x}$, and thus $F$ is an imbedding.

THEOREM 5. If $X$ is normal and strongly countably compact, then $2^{X}$ is strongly countably compact.

Proof. Let $F: 2^{X} \rightarrow 2^{\beta x}$ be defined as in the previous lemma. Then, by the previous lemma, $F$ is an imbedding. Let $\left\{K_{i}\right\}_{i=1}^{\infty}$ be a countable subset of $2^{X}$. Let $B$ be the closure of $\left\{F\left(K_{i}\right)\right\}_{i=1}^{\infty}$ in $2^{\beta X}$ and $\mathcal{C}$ the closure of $\left\{K_{i}\right\}_{i=1}^{\infty}$ in $2^{X}$. Since $F$ is an imbedding, $F(\mathbb{C})=B \cap F\left(2^{X}\right)$. If we can show that $B C F\left(2^{x}\right)$, then $F(\mathcal{C})=B$ and thus $\mathbb{C}$ will be compact since $B$ is. Thus it will be sufficient to prove that $B \subset F\left(2^{X}\right)$. 
Let $K^{*}$ be any element of $\leftrightarrow$ in $2^{\beta X}$, and let $\mathrm{Cl}_{\beta X} K_{i_{\alpha}}$ be a net converging to $K^{*}$ in $2^{\beta X}$. Let $K=K^{*} \cap X$. Now suppose that $x \in K^{*}-\mathrm{Cl}_{\beta X} K$. Then let $x \in U$ with $U$ open in $\beta X$ with $\left[\mathrm{Cl}_{\beta X} U\right] \cap\left[\mathrm{Cl}_{\beta X} K\right]=\varnothing$. Since $\mathrm{Cl}_{\beta X} K_{i_{\alpha}} \rightarrow K^{*}$, there must be a $\gamma$ such that, for $\alpha \geqq \gamma,\left[\mathrm{Cl}_{\beta X} K_{i_{\alpha}}\right]$ $\cap U \neq \varnothing$. Thus $K_{i_{\alpha}} \cap U \neq \varnothing$ for $\alpha \geqq \gamma$. Let $A=\left\{i: K_{i} \cap U \neq \varnothing\right\}$. Let $a_{i} \in K_{i} \cap U$ for each $i \in A$. Let $B=\mathrm{Cl}_{X}\left\{a_{i}: i \in A\right\}$. Then $B$ is compact by the strong countable compactness of $X$. Therefore there is a subnet of $a_{i_{\alpha}}$ converging to some $a \in B$. One can easily show that $a \in K^{*}$ and thus $a \in K^{*} \cap X=K$. But also $a \in \mathrm{Cl}_{\beta X} U$ and $\left[\mathrm{Cl}_{\beta X} U\right] \cap K \neq \varnothing$, a contradiction. Therefore we must have that $K^{*}=\mathrm{Cl}_{\beta X} K$. Thus $K^{*} \in F\left(2^{X}\right)$ and $B \subset F\left(2^{X}\right)$.

It would be interesting to know if in Theorem 5 the assumption of normality can be reduced. At any rate, the theorem is sufficient for the next application.

EXAMPLE. Let $X$ be a noncompact countably compact ordinal space. Then $X$ is strongly countably compact and normal. Thus $2^{X}$ is strongly countably compact. Thus we have an example of a noncompact space $X$ for which $2^{X}$ is countably compact and hence pseudocompact.

EXAMPLE. For each integer $n$ greater than 1, Frolík [3] has given an example of a space $X$ such that $X^{n-1}$ is countably compact and $X^{n}$ is not pseudocompact. In the examples $X^{n}$ fails to be pseudocompact by containing an open and closed copy of the integers $N$. Let $p: X^{n} \rightarrow \mathcal{F}_{n}(X)$ be defined by $p\left(\left(x_{1}, \cdots, x_{n}\right)\right)=\left\{x_{1}, \cdots, x_{n}\right\}$. Then $p$ is closed and continuous [4]. Now let $N$ be an open and closed copy of the integers in $X^{n}$. Then $p(N)$ is closed in $F_{n}(X)$ and thus is closed in $2^{x}$. Now for $\left(x_{1}, \cdots, x_{n}\right)$ an isolated point in $X^{n}$, we have $\left\{x_{1}, \cdots, x_{n}\right\}$ is also an isolated point in $2^{x}$. Thus $p(N)$ is open and closed in $2^{x}$. Since $p$ is finite to one, $p(N)$ is infinite and $2^{x}$ contains an open and closed copy of the integers. Thus $2^{x}$ is nonpseudocompact. Thus Frolík's examples give examples of countably compact completely regular spaces whose hyperspaces are not pseudocompact.

The author would like to express his gratitude to the referee for his helpful suggestions for improving the paper.

ADDED IN PROOF. Assuming the continuum hypothesis the author has been able to show that $2^{X}$ is normal if and only if $X$ is compact.

\section{REFERENCES}

1. G. Aquaro, "Point countable open coverings and countably compact spaces" in General topology and its relations to modern analysis and algebra. II (Proc. Second Prague Topological Sympos., 1966) Academia, Prague, 1967, pp. 39-41. MR 38 \#654. 
2. J. Dugundji, Topology, Allyn and Bacon, Boston, Mass., 1966. MR 33 \#1824.

3. Z. Frolík, Sums of ultrafilters, Bull. Amer. Math. Soc. 73 (1967), 87-91. MR $34 \# 3525$.

4. T. Ganea, Symmetrische Potenzen topologischer Räume, Math. Nachr. 11 (1954), 305-316. MR 16, 503.

5. L. Gillman and M. Jerison, Rings of continuous functions, Van Nostrand, Princeton, N. J., 1960. MR 22 \#6994.

6. V. M. Ivanova, On the theory of the space of subsets, Dokl. Akad. Nauk SSSR 101 (1955), 601-603. MR 16, 1041. (Russian)

7. K. Kuratowski, Topology. Vol. I, Academic Press, New York, 1966, pp. 160182. MR $36 \# 840$.

8. E. Michael, Topologies on spaces of subsets, Trans. Amer. Math. Soc. 71 (1951), 152-182. MR 13, 54.

9. H. Wallman, Lattices and topological spaces, Ann. of Math. (2) 39 (1938), 112126.

UNIVERSITY OF FloRIDA 